\title{
Bildungsfinanzierung und Bildungsrenditen
}

\section{Bernhard A. Weber}

Der folgende Aufsatz befasst sich mit dem Zusammenhang zwischen der privaten bzw. öffentlichen Finanzierung von Bildung und Bildungsrenditen. Dazu wird in einem ersten Teil ein kurzer Einblick in die Grundlagen der Humankapitaltheorie vermittelt, wobei die Konzepte von privaten, fiskalischen und öffentlichen Bildungsrenditen erläutert werden. In einem zweiten Teil wird der theoretische Zusammenhang zwischen Bildungsfinanzierung und Bildungsrenditen dargestellt. Dabei werden sowohl komparativ-statische als auch dynamische Überlegungen gemacht. In Teil drei werden Schätzungen privater, fiskalischer und sozialer Bildungsrenditen für Männer in der Schweiz präsentiert. Anhand dieser Daten wird illustriert, welche Wirkung eine vollständige Privatisierung bzw. eine vollständig öffentlichen Finanzierung direkter Bildungskosten auf Bildungsrenditen hätte (komparativ-statische Überlegungen). Dynamische Auswirkungen solcher Politikänderungen werden anhand internationaler, empirischer Erkenntnisse eingeschätzt. Der Aufsatz schliesst mit Schlussfolgerungen sowie einem Überblick über laufende und einem Ausblick auf neue Forschungsfelder.

\section{Einleitung}

Bildung ist in Industrieländern gemessen am finanziellen Engagement eine der wichtigsten Staatsaufgaben. In der Schweiz wendet die öffentliche Hand rund 5.5\% des Bruttoinlandproduktes für Bildung auf. Die obligatorische Schulbildung ist ein Beispiel für eine vollständig staatlich finanzierte Schulbildung. Auf der anderen Seite gibt es Bildungsformen, bei denen die gesamten Kosten durch Individuen oder private Institutionen getragen werden und der Staat nur eine untergeordnete Rolle spielt, wie beispielsweise ein privater, in der Freizeit besuchter Weiterbildungskurs. Dazwischen gibt es viele Formen von Bildung, und hier liegt auch die Mehrheit der Ausbildungsgänge in der Schweiz, welche durch öffentliche und private Quellen gemeinsam finanziert werden. Die Frage, welches die optimale Mischung privater und öffentlicher Finanzierung ist, ist in der bildungsökonomischen Forschung zentral. 


\section{Humankaptialtheorie und Bildungsrenditen}

\section{Grundidee der Humankapitaltheorie}

Die Humankapitaltheorie bildet die Grundlage zur Berechnung von Bildungsrenditen. Ihre zentrale Idee besteht darin, dass Menschen ihre Fähigkeiten weiterentwickeln können, indem sie in sich selber investieren. Nach humankapitaltheoretischer Auffassung steigern alle Arten der Bildung wie Schulbildung, Aus- und Weiterbildung, aber auch das «on the job training» die Produktivität einer Arbeitskraft, was sich auf einem kompetitiven Arbeitsmarkt in einem höheren Lohnsatz niederschlägt. Der bildungsbedingte Lohnvorteil, die sogenannte Ausbildungsprämie, kann dabei als die Verzinsung des Humankapitals aufgefasst werden. Als Investitionskosten sind auf der anderen Seite als Opportunitätskosten in Form von entgangenem Lohneinkommen sowie als direkte Ausbildungskosten zu veranschlagen. Bildungsrenditen geben darüber Auskunft, inwieweit der zu erwartende Nutzen einer Bildungsinvestition die Kosten zu kompensieren vermag. Es gibt verschiedene Ansätze zur Berechnung von Bildungsrenditen, wobei die Wahl der Schätzmethode von der Fragestellung und der Verfügbarkeit geeigneter Daten abhängt. Letztlich beruhen jedoch alle Methoden auf Kosten-Nutzen Überlegungen (vgl. z.B. Blundell, Dearden \& Sianesi, 2001; Card, 1999; Psacharopoulos, 1987; Wolter, 2002).

Das Investitionsproblem für einen bestimmten Ausbildungsgang $h$ lässt sich wie in Gleichung (1) darstellen. Auf der linken Seite stehen die Investitionskosten als Summe direkter Bildungskosten $C_{h}$ (Bildungsausgaben) und Opportunitätskosten $W_{h-1}$ (Lohneinkommen einer Person auf dem Ausgangsbildungsniveau $h-1) . S_{h}$ repräsentiert dabei die Ausbildungsdauer in Anzahl Jahren. Rechterhand ist der Ertrag der Bildungsinvestition als Summe der Lohndifferenz dargestellt, welche Personen mit Ausbildungsniveau $h$ gegenüber Personen mit Bildungsniveau $h-1$ vom Schulabschluss bis zur Pensionierung $P$ erzielen. Um dem intertemporalen Charakter der Investitionsentscheidung gerecht zu werden, werden Kosten und Erträge mit einem Zinssatz $r$ abdiskontiert. Die Bildungsrendite entspricht dabei demjenigen, sog. internen Zinssatz, welcher die Gegenwartswerte von Kosten und Erträgen gleichsetzt (Ertragsratenansatz).

\section{$\sum_{i}^{\prime}\left(c_{h}+W_{-t}\right) \cdot(I+\nu)^{-1}-\sum_{n+1}^{\infty}\left(W_{1}-W_{L-t}\right)(l+\nu)^{-t}$}

Die Bildungsrendite sagt für sich genommen noch nichts darüber aus, ob eine Investition sich lohnt oder nicht. Dazu ist die Gegenüberstellung mit einem sogenannten Kalkulationszinssatz nötig. Theoretisch handelt es sich dabei um einen Kapitalmarktzinssatz, zu dem jederzeit und in beliebiger Höhe Finanzinvestitionen getätigt und Kredite aufgenommen werden können. Er dient damit als Indikator für die erreichbare Alternativverzinsung. Eine Investition ist 
dann durchzuführen, wenn ihr interner Zinssatz grösser als der vorgegebene Kalkulationszinssatz ist. Bei Vorliegen verschiedener Alternativen ist diejenige mit der höchsten Ertragsrate den anderen vorzuziehen.

\section{Private, soziale und fiskalische Bildungsrenditen}

Eine erste wichtige Unterscheidung bei der Berechnung von Bildungsrenditen betrifft die Frage, aus welcher Sicht eine Bildungsinvestition bewertet wird. Als Investoren in Bildung kommen Individuen oder Unternehmen oder aber der Staat in Frage, wobei die verschiedenen Akteure unterschiedliche Kosten und Erträge ins Kalkül einbeziehen werden. In der Literatur werden u.a. drei Typen von Bildungsrenditen unterschieden, deren Bedeutung anhand von Abbildung 1 veranschaulicht werden kann (Alsalam \& Conley, 1995).

Ausgangspunkt der Überlegungen bilden Brutto-Lebenseinkommensprofile von Personen mit unterschiedlichen Bildungsabschlüssen $\left(W_{h}, W_{h-1}\right)$. Wichtig für die Unterscheidung einer öffentlichen und einer privaten Sichtweise ist auch das Steuersystem, welches hier anhand eines einkommensabhängigen Steuersatzes $\tau$ dargestellt wird. Die Netto-Lebenseinkommensprofile ergeben sich zu $\mathrm{W}_{\mathrm{h}} \cdot\left(1-\tau_{\mathrm{Wh}}\right)$ bzw. $\mathrm{W}_{\mathrm{h}-1} \cdot\left(1-\tau_{\mathrm{Wh}-1}\right)$. Der Beginn einer Ausbildung wird als Zeitpunkt 0 definiert, $S_{h}$ bezeichnet die Ausbildungsdauer in Jahren und P den Zeitpunkt der Pensionierung. Nach unten abgetragen sind die direkten Ausbildungskosten, wobei hier zwischen privaten $\left(\mathrm{C}_{\mathrm{h}} \mathrm{pr}\right)$ und öffentlichen Kosten $\left(\mathrm{C}_{\mathrm{h}} \mathrm{pu}\right)$ unterschieden wird. In Analogie zu Gleichung (1) lassen sich damit sog. private, fiskalische und soziale Bildungsrenditen berechnen.

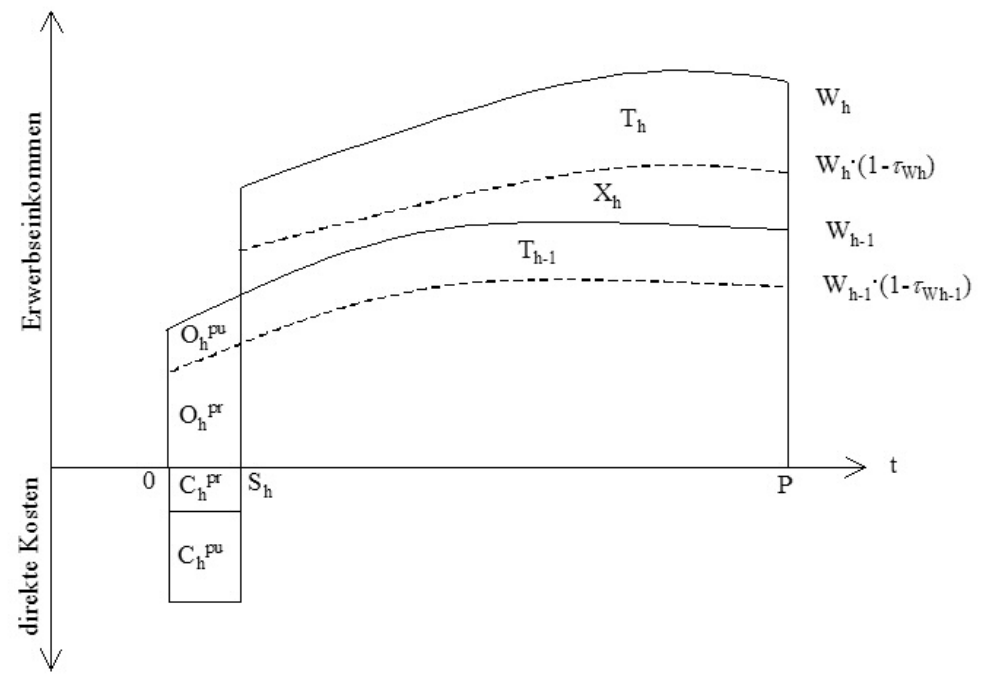

Abbildung 1: Private, öfentliche und fiskalische Bildungsrenditen im KostenNutzen Ansatz (Quelle: eigene Darstellung) 
Private Renditen: Bei der Berechnung privater Bildungsrenditen werden direkte private Kosten (Fläche $\mathrm{C}_{\mathrm{h}}{ }^{\mathrm{pr}}$ ) und private Opportunitätskosten in Form des entgangenen Netto-Erwerbseinkommens während der Ausbildung (Fläche $\mathrm{O}_{\mathrm{h}} \mathrm{pr}^{\mathrm{r}}$ ) den privaten Erträgen, d.h. dem Nettoeinkommensvorteil gegenüber dem nächst-tieferen Ausbildungsgang (Fläche $\mathrm{T}_{\mathrm{h}-1}+\mathrm{X}_{\mathrm{h}}$ ) gegenübergestellt.

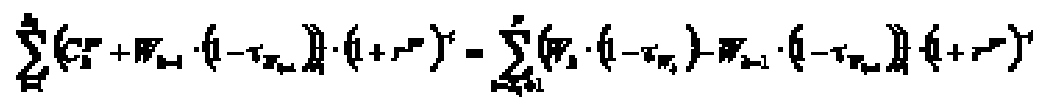

Private Bildungsertragsraten zeigen, wie lohnend zusätzliche Investitionen in Bildung aus der Sicht eines einzelnen Investors sind. Private Renditen sind nach humankapitaltheoretischer Auffassung der Anreizmechanismus für die Bildungsnachfrage und leisten damit einen Beitrag zur Erklärung der individuellen Bildungsnachfrage.

Soziale Renditen: Die gesamten direkten und indirekten Kosten (Fläche $\mathrm{C}_{\mathrm{h}} \mathrm{pu}_{+}$ $\mathrm{C}_{\mathrm{h}} \mathrm{pr}^{\mathrm{pr}} \mathrm{O}_{\mathrm{h}}{ }^{\mathrm{pr}}+\mathrm{O}_{\mathrm{h}}{ }^{\mathrm{pu}}$ ) werden den Bruttoeinkommensdifferenzen (Fläche $\mathrm{X}_{\mathrm{h}}+$ $\mathrm{T}_{\mathrm{h}}$ ) gegenübergestellt.

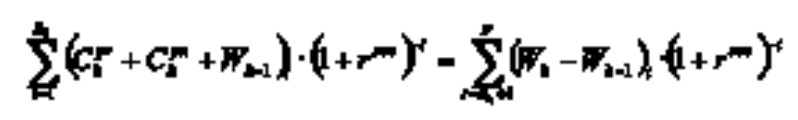

Soziale Bildungsrenditen geben an, wie rentabel Investitionen ins Bildungswesen für die Gesamtwirtschaft sind. Sie können damit beispielsweise als Planungsinstrument der Bildungspolitik dienen. Gleichung (3) gibt dabei eine sehr eng definierte soziale Bildungsrendite wieder, da nicht alle Umverteilungswirkungen des Fiskalsystems berücksichtigt und positive externe Effekte ${ }^{1}$ vernachlässigt werden (vgl. Sianesi \& Van Reenen, 2002 für einen Überblick über die makroökonomische Literatur zum Thema Bildungsrenditen).

Fiskalische Renditen: Die öffentlichen direkten und indirekten Kosten (Fläche $\mathrm{C}_{\mathrm{h}}{ }^{\mathrm{pu}}+\mathrm{O}_{\mathrm{h}}{ }^{\mathrm{pu}}$ ) werden mit den zusätzlichen Steuereinnahmen (Fläche $\mathrm{T}_{\mathrm{h}}-\mathrm{T}_{\mathrm{h}-1}$ ) verglichen.

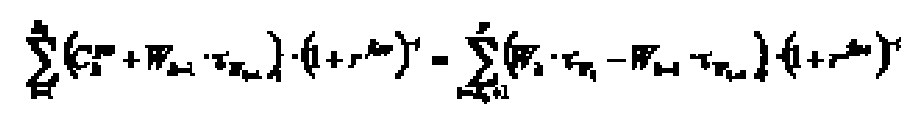

Fiskalische Bildungsrenditen zeigen an, inwieweit öffentliche Bildungsausgaben über zukünftig höhere Steuerabgaben der Investoren abgegolten werden. Sie sind damit ein Indikator zur Verteilungswirkung steuerfinanzierter Bildung. Auch hier führt die Vernachlässigung gewisser weiterer Umverteilungswirkungen und von Externalitäten zu einer Unterschätzung der wahren Rendite. 


\section{Der Zusammenhang $z w i s c h e n$ Finanzierungsformen und Bildungsrenditen}

\section{überblick}

Die Kernfragestellung dieses Beitrages lautet, welche Zusammenhänge zwischen verschiedenen Arten der Bildungsfinanzierung und Bildungsrenditen bestehen. Der Beitrag konzentriert sich dabei auf die Unterscheidung von privater vs. öffentlicher Finanzierung von Bildung. In Abbildung 2 sind die wichtigsten Einflussfaktoren privater, sozialer und fiskalischer Bildungsrenditen und deren wechselseitigen Abhängigkeiten in einem Überblick wiedergegeben.

Abbildung 2: Einflussgrössen von Bildungsrenditen (Quelle: eigene Darstellung)

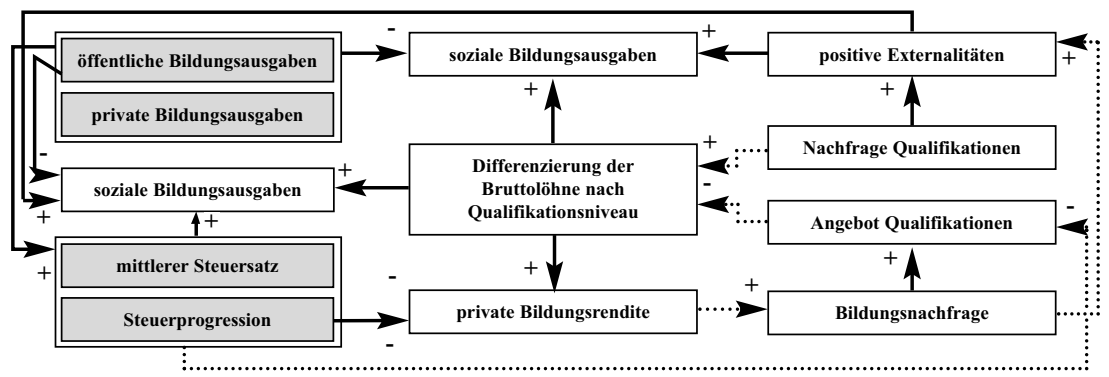

\section{Komparativ statische überlegungen}

Zentrale Einflussgrösse von Bildungsrenditen - und daher im Zentrum des Diagramms dargestellt - ist die Differenzierung von Bruttolöhnen nach Qualifikationsniveaus. Sie ist sowohl kosten- (Opportunitätskosten), als auch ertragsseitig (qualifikationsabhängiger Lohnvorteil) die wichtigste Einflussgrösse.

Private Bildungsrenditen hängen neben relativen Löhnen zudem vom System der Bildungsfinanzierung ab, d.h. einerseits vom privat finanzierten Anteil an den direkten Bildungskosten und von der Ausgestaltung des Steuersystems. Wie sich zeigen lässt, ist beim Steuerystem insbesondere die Progression von Bedeutung. Ein progressives Steuersystem kommt einer impliziten Besteuerung auf Humankapital gleich, da höhere Einkommen und damit Erträge aus Humankapital einem höheren Steuersatz unterliegen. Ein proportionales Steuersystem verzerrt private Bildungsrenditen dagegen nur geringfügig, da Bildungskosten und -erträge um den gleichen Prozentsatz verringert werden (wenn man von direkten Bildungskosten absieht). Eine öffentliche Bildungsfinanzierung und ein progressives Steuersystem haben auf private Bildungsrenditen also gegenläufige Wirkung, und es ist eine empirische Frage, ob vom Gesamtsystem der Bildungsfinanzierung unter dem Strich eher positive oder negative Anreize auf Humankapitalinvestitionen ausgehen. Neben möglichen gegenläufigen Effekten ist auch die enge logische Verknüpfung von Steuersystem und steuerfinanzierten Bil- 
dungsausgaben zu beachten, denn eine Erhöhung des privaten Anteils an den Bildungsausgaben setzt Steuermittel frei. Die Art und Weise, wie diese den Steuerzahlern rückerstattet werden, kann private Bildungsrenditen beeinflussen.

Etwas einfacher verhält es sich mit sozialen Renditen. Sie sind von relativen Löhnen, den Gesamtausgaben für Bildung und allfälligen positiven Externalitäten beeinflusst. Die Frage, wie das Steuersystem ausgestaltet ist und zu welchen Teilen Bildung privat oder öffentlich finanziert wird, ist für die Höhe sozialer Bildungsrenditen irrelevant, solange davon die Höhe der Gesamtausgaben und das Verhalten der verschiedenen Akteure unbeeinflusst bleibt. Könnte ein bestimmtes Bildungsfinanzierungssystem allerdings beispielsweise eine höhere Effizienz für sich beanspruchen, d.h. die gleiche Bildungsleistung zu geringeren Kosten bereitstellen, wäre ein positiver Einfluss auf die soziale Bildungsrendite zu erwarten (vgl. Wolter, 2001 zur Wirkung der Bildungsfinanzierung auf die Effizienz der Bildung). Die grosse Unbekannte bei der Schätzung sozialer Bildungsrenditen sind die vermuteten positiven Externalitäten, deren Ausmass bis heute weitgehend unbekannt ist.

Fiskalische Renditen hängen unter anderem ebenfalls von relativen Löhnen nach Qualifikationen ab. Grösser noch als bei privaten Bildungsrenditen ist hier allerdings der Einfluss des Steuer- und Bildungsfinanzierungssystems. Je höher öffentliche Bildungsausgaben sind, desto geringer fallen - unter sonst gleichen Bedingungen - die fiskalischen Bildungsrenditen aus. Eine Privatisierung direkter Ausbildungskosten wäre dagegen mit einer Erhöhung fiskalischer Bildungsrenditen verbunden. Ähnlich wie bei privaten Bildungsrenditen ist auch bei fiskalischen Bildungsrenditen weniger die Höhe des durchschnittlichen Steuersatzes als vielmehr die Steuerprogression von Bedeutung. In einem progressiv ausgestalteten Steuersystem werden die niedrigen Lohneinkommen am Anfang der Berufslaufbahn steuerlich unterdurchschnittlich belastet. Die Steuerausfälle von nicht erwerbstätigen, jungen Studierenden sind entsprechend gering. Umgekehrt werden höhere Lohneinkommen nach Studienabschluss in progressiven Steuersystemen mit höheren Steuersätzen belastet, wodurch Humankapitalerträge auch für den Fiskus zusätzliche Erträge abwerfen. Fiskalische Bildungsrenditen sind daher - unter sonst gleichen Bedingungen - um so höher, je progressiver ein Steuersystem ausgestaltet ist.

\section{Dynamische überlegungen}

In der bisherigen Beschreibung wurden Verhaltensänderungen der Akteure ausgeblendet. Solche dynamischen Effekte sind in Abbildung 2 mit gestrichelten Pfeilen eingetragen. Zentral ist in unserem Zusammenhang, dass qualifikationsspezifische Bruttolöhne als Ergebnis des Arbeitsmarktes zu interpretieren sind, womit sie vom Angebot an und der Nachfrage nach den entsprechenden Qualifikationen abhängen.

Die Nachfrage nach Qualifikationen wird in Abbildung 2 als exogen dargestellt, eine Annahme, die sich in einer kurzfristigen Perspektive durchaus recht- 
fertigen lässt. Längerfristig dürfte die Nachfrage allerdings auch mit vom Angebot an qualifizierten Arbeitskräften abhängen, wenn man davon ausgeht, dass Arbeitskräfte im Vergleich zu Kapital wenig mobil sind und ein grosses Angebot qualifizierter Arbeitskräfte in einer Region zur Ansiedlung von Unternehmen mit einer hohen Nachfrage nach solchen Arbeitskräften führen kann. ${ }^{2}$ Eine interessante These vertritt beispielsweise auch Acemoglu (2001). Er argumentiert, dass eine grosse Verfügbarkeit qualifizierter Arbeitskräfte den technologischen Fortschritt in eine Richtung lenken kann, welche die Nachfrage nach qualifizierten Arbeitskräften steigert. Der viel zitierte «skill-bias» des technologischen Fortschritts wäre damit durch ein steigendes Angebot an qualifizierten Arbeitskräften induziert.

Das Angebot an Qualifikationen auf dem Arbeitsmarkt hängt im hier dargestellten Modell von der Bildungsnachfrage und damit indirekt von den privaten Bildungsrenditen ab: Je höher private Bildungsrenditen sind, desto grösser ist - ceteris paribus - die Bildungsnachfrage und desto mehr qualifizierte Arbeitskräfte treten - mit ein paar Jahren Verzögerung - auf dem Arbeitsmarkt. Natürlich hängt die Bildungsnachfrage von vielen weiteren, beispielsweise institutionellen oder kulturellen Faktoren ab, welche im Diagramm nicht dargestellt sind und implizit als konstant und vom Finanzierungsmodus unabhängig betrachtet werden.

Das Steuersystem wird in Abbildung 2 ebenfalls als Einflussfaktor des Arbeitsangebots dargestellt, da hohe Grenzsteuersätze negative Arbeitsanreize ausüben können. Auch das Arbeitsangebot hängt von weiteren, im Diagramm ausgeblendeten Faktoren ab. Von Bedeutung sind beispielsweise Migrationsbewegungen, Veränderungen im Partizipationsverhalten z.B. der Frauen und demografische Faktoren, wie z.B. die Grösse und Qualifikationsstruktur der in den Arbeitsmarkt neu eintretenden und der daraus austretenden Kohorten.

Über den Einfluss privater Bildungsrenditen auf die Bildungsnachfrage, geht längerfristig ein Rückkoppelungseffekt auf die qualifikationsabhängigen Lohndifferenz und damit letztlich auf die Höhe sozialer, fiskalischer und privater Bildungsrenditen aus. Dieser Rückkoppelungseffekt kann über ein Modell verdeutlicht werden, welche die Interaktion zwischen Bildungs- und Arbeitsmarkt aufzeigt.

\section{Allgemeine Gleichgewichtsüberlegungen an einem Marktmodel l}

Üblicherweise geht man in Modellen der Bildungsnachfrage wie in Abbildung 3 im Quadranten I von einer fallenden, aggregierten Nachfragekurve nach Bildungsabschlüssen aus $\left(\mathrm{ND}_{\mathrm{h}}\right)$. Diese lässt sich aus der Annahme herleiten, dass Bildungsrenditen von Person zu Person, beispielsweise in Abhängigkeit ihrer Begabung, variieren. Unter dieser Annahme werden um so mehr Jugendliche ein Diplom einer Bildungsstufe h erwerben wollen, je geringer der Zinnsatz für alternative Anlagen ist. Mit anderen Worten werden bei tiefem Zinsniveau auch Personen mit relativ geringen erwarteten privaten Bildungsrenditen Bildungsinvestitionen tätigen. 
Die Angebotskurve $A_{K}$ repräsentiert demgegenüber das Angebot alternativer Kapitalanlagen. Rationale Bildungsnachfrager werden in einen Ausbildungsgang $h$ investieren, solange die erwartete private Bildungsrendite über dem Zinssatz für alternative Kapitalanlagen liegt, da sie damit den Gegenwartswert ihres Lebenseinkommens maximieren. Eine steigende Angebotskurve ergibt sich daraus, dass Alternativzinssätze von Person zu Person variieren. Personen, welche ihre Ausbildung mit Eigenmitteln finanzieren, haben beispielsweise einen tieferen Alternativzinssatz (z.B. den Spargeldzinssatz) als Personen, welche eine Ausbildung fremdfinanzieren müssen (z.B. Zinssatz für die Aufnahme eines relativ riskanten Kredites). Neben den Kapitalmarktbedingungen kann die Angebotskurve zudem durch individuelle Zeitpräferenzen beeinflusst sein: Während es gewisse Personen bei einem bestimmten Kapitalmarktzinssatz vorziehen, Geld zu sparen und damit Konsum auf spätere Perioden zu verschieben, würden andere beim gleichen Zinssatz bereits einen Kredit aufnehmen, um in der Gegenwart mehr konsumieren zu können (Becker \& Mulligan, 1997). Damit finden wir links unten auf der Angebotskurve für Kapital vor allem Personen, welche selber Geldmittel zur Verfügung haben und/oder die eine relativ hohe Präferenz für zukünftigen Konsum haben. Oben rechts auf der Angebotskurve finden wir dagegen Leute, welche relativ teuere Bildungskredite aufnehmen müssten und/oder die eine hohe Präferenz für den Konsum in der Gegenwart aufweisen.

Abbildung 3: Ein Marktmodell von Nachfrage und Angebot nach Qualifikationen (Quelle: eigene Darstellung, in Anlehnung an Blaug [1970], S. 179)

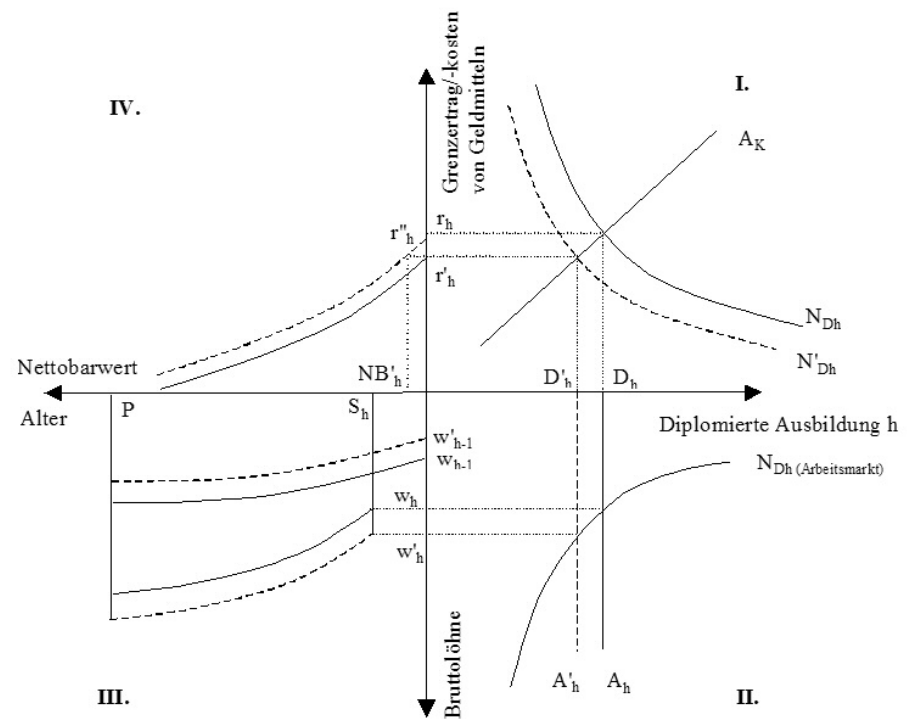


In Quadrant I, dem Bildungsmarkt-Modell, wird die Wirkung einer vollständigen Privatisierung direkter Bildungskosten simuliert, welche zu einer Verschiebung der Nachfragekurve nach ${ }^{\prime}{ }_{D h}$ führt. Private Bildungsrenditen der Ausbildung h sinken bei steigenden privaten Kosten - ceteris paribus. Die Absolventenzahl sinkt auf $D^{\prime}{ }_{h}$, private marginale Bildungsrenditen auf $r^{\prime}{ }_{h}$. Soziale Bildungsrenditen bleiben zunächst unbeeinflusst, da sich totale Bildungskosten und Lohndifferentiale bislang nicht verändert haben. Fiskalische Renditen steigen an, da sich die öffentlichen Bildungskosten nun auf den Steuerausfall von Personen in Ausbildung reduzieren.

Im Quadranten II ist dargestellt, wie der Arbeitsmarkt auf die - mit einigen Jahren Verzögerung eintretende - Verminderung des Angebots an Absolventinnen und Absolventen reagiert. Unterstellt wird dabei eine mit zunehmenden Bruttolöhnen fallende, über die Zeit konstante Arbeitsnachfragefunktion. Das Arbeitsangebot von Neuabsolventinnen und -absolventen ist der Einfachheit halber als vollkommen inelastisch, also in Form einer vertikalen Angebotskurve dargestellt. ${ }^{3}$ Die Verknappung des Angebots an qualifizierten Arbeitskräften auf $\mathrm{D}^{\prime}{ }_{\mathrm{h}}$ schlägt sich in diesem Fall in einer Erhöhung der Einstiegslöhne von $w_{h}$ nach $w_{h}^{\prime}$ nieder. Das Angebot an Absolventinnen und Absolventen der Bildungsstufe h-1 (in der Grafik nicht dargestellt) erhöht sich spiegelbildlich zum Angebot höherer Qualifikationen, womit sich die Einstiegslöhne von $\mathrm{w}_{\mathrm{h}-1}$ auf $\mathrm{w}_{\mathrm{h}-1}$ verringern.

In Quadrant III sind die Konsequenzen für die Lebenseinkommensbetrachtung dargestellt. Geht man bei der Privatisierung der Bildungsausgaben von einer permanenten Politikänderung aus, dürfte sich das gesamte Lebenseinkommensprofil mit Bildungsabschluss $h$ nach oben und mit Abschluss $h-1$ nach unten verschieben. Die Verknappung höherer Qualifikationen führt damit längerfristig wieder zu einer Erhöhung der Lohndifferenzen.

In Quadrant IV ist die Auswirkung dieser Veränderung auf den Nettobarwert einer Bildungsinvestition dargestellt. Der Nettobarwert ist dabei als Funktion des Alternativzinssatzes (vertikale Achse) abgetragen. Als Referenzsituation ist mit der ausgezogenen Kurve die Situation mit privatisierten Bildungsausgaben aber ohne Veränderung der relativen Löhne dargestellt. Dabei beträgt der Nettobarwert Null, wenn der Alternativzinssatz der Bildungsrendite $r_{h}{ }_{h}$ entspricht. Steigt nun die relative Lohndifferenz, so verschiebt sich die Kurve nach links (gestrichelte Linie), da der Nettobarwert der Bildungsinvestition bei gegebenem Zinssatz steigt. Wie die Grafik zeigt sind nun Bildungsinvestitionen bei einem Alternativzinssatz von $r^{\prime}{ }_{h}$ wieder attraktiv: es resultiert ein positiver Nettobarwert. Der interne Zinssatz, welcher den Gegenwartswert von Kosten und Erträgen der Bildungsinvestition gleichsetzt beträgt nun $\mathrm{r}^{\prime}{ }_{\mathrm{h}}$ und liegt zwischen $\mathrm{r}_{\mathrm{h}}$ und $\mathrm{r}_{\mathrm{h}}{ }_{\mathrm{h}}$.

Als Folge davon verschiebt sich die Nachfragekurve in Quadrant I wieder ein Stück weit nach rechts, bis sie die Kapitalangebotskurve beim Zinssatz r" schneidet. Auch diese Verschiebung hat nun analog zum ersten Durchgang dynamische Anpassungen zur Folge. Im Endeffekt wird sich die Nachfragekurve 
zwischen $\mathrm{N}_{\text {Dh }}$ und $\mathrm{N}^{\prime}$ Dh und die private Bildungsrendite zwischen $\mathrm{r}_{\mathrm{h}}$ und $\mathrm{r}_{\mathrm{h}}$ einpendeln. Die Frage, wo die Kurven und die neuen Gleichgewichtswerte genau zu liegen kommen, hängt, wie die Analyse gezeigt hat, von einer ganzen Reihe von Faktoren ab.

Im Endeffekt dürfte eine Privatisierung von Ausbildungskosten jedoch

- zu einer Verringerung der Bildungsnachfrage,

- einem Rückgang der privaten Bildungsrendite und

- einer Erhöhung der qualifikationsbedingten Lohnprämie und damit

- einer Erhöhung sozialer und fiskalischer Bildungsrenditen führen.

Die negative Wirkung einer Privatisierung von Ausbildungskosten auf private Bildungsrenditen ist gemäss unserem Modell um so grösser,

- je inelastischer das Angebot an alternativen Kapitalanlagen ist $\left(A_{K}\right.$ steiler),

- je grösser der öffentliche Anteil am Total der direkten und indirekten Bildungskosten im Referenzzustand ist (Links-Verschiebung von $\mathrm{N}_{\mathrm{Dh}}$ stärker) und

- je elastischer die Nachfrage nach qualifizierten Arbeitskräften ist $\left(\mathrm{N}_{\text {Dh(Arbeitsmarkt) }}\right.$ flacher).

Die Wirkung einer Privatisierung von Bildungskosten auf soziale Bildungsrenditen hängt von der Reaktion der relativen Löhne ab. Die Lohnspreizung und damit die Erhöhung der sozialen Bildungsrendite fällt dabei um so grösser aus,

- je elastischer das Angebot an alternativen Kapitalanlagen ist ( $A_{K}$ flacher),

- je grösser der öffentliche Anteil am Total der Bildungskosten im Referenzzustand ist (Links-Verschiebung von $\mathrm{N}_{\mathrm{Dh}}$ stärker) und

- je inelastischer die Nachfrage nach qualifizierten Arbeitskräften ist $\left(\mathrm{N}_{\text {Dh(Arbeitsmarkt) }}\right.$ steiler).

Fiskalische Bildungsrenditen sind stärker von Faktoren des Bildungsfinanzierungssystems als von Marktreaktionen abhängig. Die Erhöhung fiskalischer Bildungsrenditen wird daher vor allem um so grösser ausfallen, je grösser der öffentliche Anteil am Total der Bildungskosten ursprünglich ist. Ein elastisches Kapitalangebot und eine inelastische Nachfrage nach qualifizierten Arbeitskräften können die Wirkung über eine Akzentuierung der Lohnspreizung noch verstärken.

Veränderungen von Bildungsrenditen sind im beschriebenen Modell eine Folge von Veränderungen in der Bildungsnachfrage. Die Bildungsnachfrage wird bei einer Privatisierung der Ausbildungskosten um so stärker reduziert,

- je elastischer das Angebot an alternativen Kapitalanlagen ist,

- je grösser der öffentliche Anteil am Total der Bildungskosten im Referenzzustand ist und

- je elastischer die Nachfrage nach qualifizierten Arbeitskräften ist. 
Wie die dynamischen Anpassungen ablaufen hängt neben den jeweiligen Marktbedingungen (Elastizitäten) auch davon ab, inwieweit die Marktreaktionen durch die Akteure antizipiert werden. So könnte die Reaktion der Bildungsnachfrage auf eine Privatisierung der Bildungskosten $u$. U. bereits zu Beginn geringer ausfallen, als im ersten Quadranten suggeriert wird, wenn die daraus resultierenden Steigerung der qualifikationsbedingten Lohnprämie richtig antizipiert wird.

\section{Empirische Evidenz}

Wie die bisherigen Überlegungen gezeigt haben, bestehen zwischen dem System der Bildungsfinanzierung und Bildungsrenditen vielfältige Beziehungen. Es ist damit in erster Linie eine empirische Frage, wie sich Veränderungen in der Bildungsfinanzierung auf Bildungsrenditen auswirken. Im Folgenden werden zunächst Schätzungen von Bildungsrenditen in der Schweiz präsentiert. Anhand von Simulationsberechnungen wird geschätzt, wie sich Änderungen der Finanzierung auf diese Bildungsrenditen auswirken (komparativ-statisch). Bezüglich der dynamischen Wirkungen werden empirische Ergebnisse präsentiert, welche eine Verbindung zwischen dem Bildungssystem und dem Arbeitsmarkt herstellen. Die verschiedenen, in Abbildung 3 dargestellten Zusammenhänge zwischen der Bildungsfinanzierung und der Bildungsnachfrage (1. Quadrant), dem Qualifikationsangebot und relativen Lohndifferenzen (2. Quadrant) und relativen Löhne und der Bildungsnachfrage (3. und 4. Quadrant) werden dabei einzeln behandelt.

\section{Bildungsrenditen in der Schweiz}

Die folgenden Berechnungen basieren auf dem Kosten-Nutzen Ansatz, wie am Anfang dieses Beitrages beschrieben ist. Datengrundlage für die Schätzung von Lohn-Altersprofilen ist die Schweizerische Arbeitskräfteerhebung des Jahres 2002 (Bundesamt für Statistik, 2002). Die hier präsentierte Analyse beschränkt sich auf Vollzeit erwerbstätige Männer, da die Interpretation von Lohnprofilen bei Frauen durch Teilzeitarbeit und relativ häufige Erwerbsunterbrüche erschwert ist. Lohn-Altersprofile werden mittels einer linearen Regression anhand von Querschnittsdaten für Personen mit unterschiedlichen Ausbildungen separat geschätzt. Dabei werden die folgenden sechs Ausbildungstypen unterschieden: obligatorische Schule / Anlehre (OBL), Berufsausbildung (BA), Matura (MAT), Höhere Berufsausbildung (HBA), Fachhochschule (FHS) und Universitätsstudium (UNI) . Abhängige Variable der sechs Schätzgleichungen bildet der natürliche Logarithmus des jährlichen Bruttoerwerbseinkommens $\ln (\mathrm{w})$. Als unabhängige Variablen werden eine Konstante sowie das Alter (age) und das Alter im Quadrat $\left(\mathrm{age}^{2}\right)$ verwendet. Die beiden Alter-Terme erlauben es, dem typischen konkaven Verlauf der Einkommensprofile separat für jeden Ausbil- 
dungstyp Rechnung zu tragen. Aus den Gleichungen (5a-f) lassen sich die hier verwendeten Einkommensprofile reproduzieren.

\begin{tabular}{|c|c|c|c|c|c|c|}
\hline $\ln (\mathrm{w})_{\mathrm{OBL}}$ & $10.11+$ & $0.032 \cdot$ age - & $0.036 \cdot \mathrm{age}^{2} / 100$ & $\mathrm{~N}=$ & 723 & $\mathrm{R}^{2}=0.1874$ \\
\hline $\ln (\mathrm{w})_{\mathrm{BA}}$ & $10.05+$ & $0.050 \cdot$ age - & $0.049 \cdot \mathrm{age}^{2} / 100$ & $\mathrm{~N}=$ & 3942 & $\mathrm{R}^{2}=0.2158$ \\
\hline $\ln (\mathrm{w})_{\mathrm{MAT}}$ & $9.93+$ & $0.055 \cdot$ age - & $0.045 \cdot \mathrm{age}^{2} / 100$ & $N=$ & 370 & $\mathrm{R}^{2}=0.2697$ \\
\hline $\ln (\mathrm{w})_{\mathrm{HBA}}$ & $10.12+$ & $0.057 \cdot$ age - & $0.057 \cdot \mathrm{age}^{2} / 100$ & $N=$ & 1024 & $\mathrm{R}^{2}=0.1115$ \\
\hline $\ln (\mathrm{w})_{\mathrm{FHS}}$ & $9.93+$ & $0.070 \cdot$ age - & $0.069 \cdot \mathrm{age}^{2} / 100$ & $N=$ & 623 & $R^{2}=0.1639$ \\
\hline $\ln (\mathrm{w})_{\mathrm{UNI}}$ & $9.77+$ & $0.076 \cdot$ age - & $0.070 \cdot \mathrm{age}^{2} / 100$ & $N=$ & 980 & $\mathrm{R}^{2}=0.1629$ \\
\hline
\end{tabular}

Zur Erfassung des Steuersystems wird ein Modell verwendet, welches die durchschnittliche Steuerbelastung (Gemeinde-, Staats- und Bundessteuer) von natürlichen Personen in der Schweiz in Abhängigkeit vom Bruttoerwerbseinkommen w abbildet. Die Daten stammen dabei aus der Eidgenössischen Steuerverwaltung (1999). Geschätzt wird hier der durchschnittliche Steuersatz $\tau$ für ledige Personen ohne Kinder, da keine Informationen über Einkommen und $\mathrm{Zu}$ sammensetzung der Haushalte verwendet werden. In (6) ist der empirische $\mathrm{Zu}$ sammenhang zwischen $\tau$ und w dargelegt. Unterstellt wird dabei der Einfachheit halber ein für die Schweiz homogenes Steuersystem, wobei Umverteilungswirkungen durch Arbeitslosenversicherung oder steuerfinanzierte Sozialhilfe ausgeblendet sind.

$\tau=-133.0 \cdot \mathrm{w}^{-1}+2.66 \cdot 10^{-1} \cdot \mathrm{w}-7.52 \cdot 10^{-1} \cdot \mathrm{w}^{2} \quad \mathrm{~N}=13 \quad \mathrm{R}^{2}=0.9970$

Die Annahmen bezüglich direkter privater und öffentlicher Kosten, Alter des Studienbeginns und mittlerer Ausbildungsdauer sind in Tabelle 1 wiedergegeben. Es handelt sich dabei um grobe Schätzungen, da die Werte in dieser Form nicht systematisch erhoben werden und teilweise schwer zu erheben sind. Insbesondere fehlt in den Bildungsinstitutionen meist eine Kostenrechnung, womit direkte öffentliche Kosten verschiedener Ausbildungsgänge heute nur mit Schwierigkeiten abschätzbar sind.

Tabelle 1: Annahmen bezüglich der jährlichen Kosten und der zeitlichen Abfolge von Ausbildungsgängen, Männer

\begin{tabular}{|l|c|c|c|c|c|}
\hline & BA & MAT & HBA & FHS & UNI \\
\hline Direkte private Kosten & -6000 & 1200 & 6500 & 2000 & 2000 \\
Direkte öffentliche Kosten & 8300 & 17700 & 5100 & 30000 & 20000 \\
Schuldauer & 3.5 & 4 & 2 & 3 & 4.5 \\
Alter bei Schulbeginn & 16 & 16 & 24 & 24 & 20 \\
\hline
\end{tabular}

Quellen: eigene Schätzungen, diverse ${ }^{4}$ 
Die Schätzungen privater, fiskalischer und sozialer Bildungsrenditen sind in Tabelle 2 zusammengefasst. Angesichts der unsicheren Datenbasis im Bereich der direkten Ausbildungskosten und bei der Modellierung der Umverteilungswirkung des Fiskalsystems, sind die Zahlen mit der nötigen Vorsicht zu interpretieren. In der Tabelle werden die Renditen einzelner Ausbildungsgänge im Vergleich zum nächst-tieferen Ausbildungsgang ausgewiesen.

\section{Tabelle 2: Bildungsrenditen nach Ausbildungsgängen, Männer (in \%)}

\begin{tabular}{|l|c|c|c|c|c|}
\hline & BA & MAT & HBA & FHS & UNI \\
\hline private Bildungsrendite & 8.7 & 8.1 & 5.9 & 8.5 & 5.1 \\
soziale Bildungsrendite & 8.4 & 7.0 & 6.9 & 7.3 & 4.7 \\
fiskalische Bildungsrendite & 7.7 & 5.0 & 10.6 & 5.2 & 4.0 \\
\hline
\end{tabular}

Gemäss den vorliegenden Berechnungen bewegen sich private Bildungsrenditen für Männer in der Schweiz je nach Ausbildungsgang zwischen 5.1 und 8.7\%,wobei die Berufsausbildung (BA), die Matura (MAT) und das Fachhochschulstudium (FHS) relativ höhere private Bildungsrenditen aufweisen als die höhere Berufsausbildung (HBA) und das Universitätsstudium (UNI). Die hier präsentierten Schätzungen privater Bildungsrenditen für Männer liegen insgesamt in einer ähnlichen Grössenordnung wie frühere Schätzungen für die Schweiz (vgl. Wolter \& Weber, 1999) und auch wie kürzlich erschienene Ergebnissen für zehn OECD-Länder (USA, Japan, Deutschland, Frankreich, Italien, Grossbritannien, Kanada, Dänemark, Niederlande, Schweden) (vgl. Blöndal, Feld \& Girouard, 2002). In den zehn Ländern der OECD liegen private Bildungsrenditen für Männer auf Sekundarstufe II zwischen 6.4 und 16.4\% (Ländermittel 11.3\%) und auf Tertiärstufe zwischen 6.5 und 18.5\% (Ländermittel 11.5\%).

Soziale Bildungsrenditen liegen in der Schweiz für Männer mit Ausnahme höherer Berufsausbildungen etwas unterhalb der privaten Bildungsrenditen und bewegen sich in einer Bandbreite von 4.7 bis $8.4 \%$. Die Differenz zu privaten Renditen ist ein Indikator für das Ausmass der Subventionierung eines Ausbildungsganges. Die Tatsache, dass im Falle höherer Berufsausbildungen die soziale Bildungsrendite über der privaten liegt, bedeutet, dass das Schweizer Steuersystem Investitionen in höhere Berufsausbildungen implizit steuerlich belastet. In allen anderen Fällen wird diese zusätzliche Belastung durch die öffentliche Bildungsfinanzierung kompensiert. Da bei unseren Berechnungen Externalitäten und nicht-monetäre Aspekte ausgeblendet werden, stellen die vorliegenden Schätzungen eine Untergrenze der wahren sozialen Renditen dar. Auch in den zehn durch Blöndal et al. (2002) untersuchten Ländern liegen soziale leicht unterhalb von privaten Bildungsrenditen. Auch sie verwenden dabei allerdings eine enge Definition sozialer Renditen. Die Renditeschätzungen in den zehn OECDLändern bewegen sich für Männer auf Sekundarstufe II in einem Bereich von 5.0 bis $13.2 \%$ (Ländermittel $8.9 \%$ ) und auf Tertiärstufe in einer Spannbreite 
von 6.3 bis $15.2 \%$ (Ländermittel 9.6\%).

In der Regel tiefer als private und soziale Renditen, aber immer im positiven Bereich liegen für Männer in der Schweiz die fiskalischen Renditen. Die Rendite öffentlicher Bildungsausgaben bewegt sich je nach Ausbildungsgang zwischen 4.0 und 10.6\%. Die fiskalische Rendite ist bei der höheren Berufsausbildung darum besonders hoch, weil dort der Anteil privater Finanzierung bedeutend ist. Unberücksichtigt bleiben hier Umverteilungswirkungen über das System der Sozialversicherungen, allfällige positive Externalitäten, sowie die Tatsache, dass die Arbeitsmarktpartizipation mit steigendem Bildungsstand zunimmt. Dieser letzte Punkt, welche bei freiwilliger Partizipationsentscheidung aus privater Perspektive nicht zwingend von Bedeutung ist, spielt aus Sicht der öffentlichen Finanzen natürlich eine Rolle. Empirisch ist er insbesondere bei der Schätzung von Bildungsrenditen für Frauen von Relevanz, da bei ihnen die Arbeitsmarktpartizipation stärker vom Bildungsniveau abhängt als bei Männern.

\section{Simulationsberechnungen}

Das hier verwendete Kosten-Nutzen Modell ist sehr gut dazu geeignet, die Auswirkung von Verschiebungen privater und öffentlicher Anteile in der Bildungsfinanzierung auf Bildungsrenditen zu simulieren. Anhand der folgenden Berechnungen wird gezeigt, wie sich private und fiskalische Renditen verändern, wenn die in Tabelle 1 aufgeführten direkten Kosten einer Ausbildung a) vollständig durch den Staat übernommen werden bzw. b) vollständigauf die Bildungsnachfragenden überwälzt würden. Zur Berechnung der Bildungsrenditen kommt das Modell zur Anwendung, wie es in den Gleichungen (2) bis (4) dargestellt ist. Als Vergleichsgrösse werden private und fiskalische Bildungsrenditen verwendet, welche sich aus dem heutigen Finanzierungsmodus ergeben (gemischte Finanzierung gemäss Tabelle 1). Einen interessanten Vergleichsmassstab bilden auch die sozialen Bildungsrenditen, welche von der Verteilung privater und öffentlicher Kostenübernahme unbeeinflusst sind.

Die Wirkung einer vollständig öffentlichen Bildungsfinanzierung auf private Bildungsrenditen von Männern in der Schweiz sind in Tabelle 3 wiedergegeben. Wie die Ergebnisse zeigen, erhöhen sich private Bildungsrenditen für Männer durch eine öffentliche Finanzierung nur geringfügig, um 0.2-0.7 Prozentpunkte. Umgekehrt lässt sich folgern, dass Studiengebühren und Materialkosten in ihrem heutigen Ausmass einen relativ geringen Einfluss auf private Bildungs-renditen haben, da sie einen kleinen Anteil am Total direkter und indirekter Kosten ausmachen. Am grössten ist der Einfluss einer Übernahme direkter Bildungskosten durch den Staat bei der höheren Berufsausbildung (HBA), wo heute ein erheblicher Teil der direkten Kosten von den Bildungsnachfragenden selber finanziert wird. Auch hier ist der Einfluss jedoch nicht allzu gross, da die direkten Kosten im Vergleich zu den Opportunitätskosten (Netto-Erwerbseinkommen von Arbeitskräften mit Lehrabschluss) relativ gering sind. 
Tabelle 3: Private Bildungsrenditen: Private vs. öffentliche Finanzierung direkter Bildungskosten, Männer (in \%)

\begin{tabular}{|l|c|c|c|c|c|}
\hline & BA & MAT & HBA & FHS & UNI \\
\hline private Finanzierung & 7.1 & 5.8 & 5.3 & 5.5 & 3.3 \\
gemischte Finanzierung & 8.7 & 8.1 & 5.9 & 8.5 & 5.1 \\
öffentliche Finanzierung & 9.1 & 8.4 & 6.6 & 8.8 & 5.4 \\
soziale Bildungsrendite & 8.4 & 7.0 & 6.9 & 7.3 & 4.7 \\
\hline
\end{tabular}

Betrachtet man umgekehrt eine vollständig private Finanzierung direkter Ausbildungskosten (Tabelle 4), verringern sich private Bildungsrenditen für Männer um 0.6 bis 3.0 Prozentpunkte und kommen unterhalb von sozialen Bildungsrenditen zu liegen. Die relativen Veränderungen der Bildungsrenditen betragen zwischen -10\% (HBA) und rund -35\% (FHS/UNI) und fallen um so höher aus, je bedeutender die öffentliche Finanzierung heute ist. Eine vollständige Privatisierung von Bildungskosten hätte in Kombination mit einem progressiven Steuersystem gemäss unseren Berechnungen zur Folge, dass private Anreize zur Bildungsnachfrage (sprich private Bildungsrenditen) für Männer geringer wären als sie es aus gesellschaftlicher Sicht sein sollten (d.h. als soziale Bildungsrenditen).

Tabelle 4: Fiskalische Bildungsrenditen: Private vs. öffentliche Finanzierung direkter Bildungskosten, Männer (in \%)

\begin{tabular}{|l|c|c|c|c|c|}
\hline & BA & MAT & HBA & FHS & UNI \\
\hline private Finanzierung & 17.0 & 16.4 & 15.9 & 18.8 & 13.4 \\
gemischte Finanzierung & 7.7 & 5.0 & 10.6 & 5.2 & 4.0 \\
öffentliche Finanzierung & 6.8 & 4.8 & 7.7 & 4.9 & 3.6 \\
soziale Bildungsrendite & 8.4 & 7.0 & 6.9 & 7.3 & 4.7 \\
\hline
\end{tabular}

In Tabelle 4 ist die Wirkung unterschiedlicher Finanzierungsmodi auf fiskalische Bildungsrenditen von Männern in der Schweiz ersichtlich. Dabei ist zu sehen, dass eine vollständig öffentliche Finanzierung direkter Bildungskosten fiskalische Renditen von Männern gegenüber dem heutigen Zustand (gemischte Finanzierung) nur geringfügig verringert, da der private Anteil am Total der Bildungsausgaben - mit Ausnahme der Höheren Berufsausbildung - gering ist.

Eine vollständige Privatisierung direkter Ausbildungskosten hätte demgegenüber eine markante Erhöhung fiskalischer Bildungsrenditen um den Faktor 1.5 bis 3.6 zur Folge. Sie lägen gemäss unseren Berechnungen zwischen 13.4\% (UNI) und 18.8\% (FHS). Die hohe Sensitivität fiskalischer Bildungsrenditen auf Veränderungen des Finanzierungsmodus ist damit zu erklären, dass die Bildungsausgaben auf Kostenseite deutlich stärker ins Gewicht fallen als die indirekten, fiskalischen Kosten in Form entgangener Steuererträge von Personen in Ausbildung. Die Tatsache, dass fiskalische Bildungsrenditen bei einer vollständi- 
gen Privatisierung direkter Bildungskosten um ein Vielfaches über einem marktüblichen Zinssatz zu liegen kommen, bedeutet, dass ein progressives Steuersystem ohne öffentliche Bildungsfinanzierung einer Besteuerung von $\mathrm{Hu}$ mankapitalinvestitionen gleichkommt. Anders ausgedrückt sind vollständig privat finanzierte Humankapitalinvestitionen für den Fiskus mit einem progressiven Steuertarif ein lukratives "Geschäft», welches allerdings die private Anreize zur Bildungsnachfrage schmälert.

\section{Internationale Evidenz zu Steuersystemen und Bildungs finanzierung}

Die gleichen Zusammenhänge wie in der Schweiz, finden sich auch in anderen Ländern. Wie Blöndal et al. (2002) zeigen, werden Ausbildungen auf Tertiärstufe in allen OECD-Ländern substantiell subventioniert. In den kontinentaleuropäischen Ländern beträgt der öffentliche Anteil am Total der Bildungsausgaben zwischen 75 und 100\%. In den USA soll er bei knapp über 50\%, in Japan etwas über $40 \%$ und in Korea unter $20 \%$ liegen. Wie die Untersuchung für 10 Länder davon zeigt, ist das Steuersystem gleichzeitig in den meisten Ländern progressiv ausgestaltet. Am geringsten ist die Steuerprogression interessanterweise in Japan, dem Land mit dem geringsten Anteil an öffentlicher Bildungsfinanzierung der zehn untersuchten Länder. Im Durchschnitt der zehn untersuchten Länder liegen zudem die privaten leicht über den sozialen Bildungsrenditen, was zeigt, dass über die öffentliche Finanzierung die negative Wirkung des Steuersystems vielfach kompensiert wird (Nagel \& Jaich, 2002).

Auch in der Literatur der Finanzwissenschaften finden sich viele theoretische und auch empirische Arbeiten, welche den Zusammenhang zwischen Steuersystem und Bildungsinvestitionen behandeln. Wie diese Literatur verdeutlicht, ist die Frage der Bildungsfinanzierung nicht nur mit Effizienzfragen sondern auch mit Verteilungsfragen verknüpft (Creedy, 1994). Eine interessante Frage ist dabei, wie eine bestimmte, beispielsweise politisch gewünschte Umverteilung am effizientesten bewerkstelligt werden kann. Neuere Arbeiten in diesem Gebiet zeigen, dass eine Kombination öffentlicher Bildungsfinanzierung mit einem progressiven Steuersystem einen optimalen «policy-mix» darstellen kann. Gemäss Bovenberg und Jacobs (2001) lässt sich ein grosser Teil der öffentlichen Bildungsfinanzierung in OECD-Staaten als Gegenstück zur Einkommensumverteilung über progressive Steuern rechtfertigen bzw. erklären. Dur und Teulings (2001) argumentieren ferner, dass die dynamischen Effekte einer öffentichen Bildungsfinanzierung Lohndifferentiale zwischen Qualifikationen und damit den Bedarf für Umverteilung verringern kann.

\section{Bildungsfinanzierung und Bildungsnachfrage}

Wie die Simulationsberechnungen zeigen, hätte eine vollständige Privatisierung der Bildungsfinanzierung in der heutigen Situation eine deutliche Senkung pri- 
vater Bildungsrenditen zur Folge. Entsprechend wäre, gemäss Humankapitaltheorie, bei einer Reduktion staatlicher Subventionen mit einem Rückgang der Bildungsnachfrage zu rechnen, wie dies in unserem Marktmodell in Abbildung 3 dargestellt ist.

Zur Schätzung des Einflusses einer öffentlichen bzw. privaten Bildungsfinanzierung auf die Bildungsnachfrage kommen in der empirischen Literatur verschiedene Strategien zur Anwendung. Anhand von Zeitreihen-Analysen wird untersucht, inwieweit Veränderungen der Bildungsnachfrage über die Zeit durch Änderungen im Finanzierungsmodus und andere ökonomische Variablen zu erklären sind. Auch mit Querschnittsvergleichen zwischen Ländern oder Gliedstaaten eines Landes mit unterschiedlichen Finanzierungssystemen, kann die Wirkung verschiedener Finanzierungsarten auf die Bildungsnachfrage getestet werden. Schliesslich ist es auch möglich, eine Querschnitts- mit einer Zeitreihenanalyse in einer sogenannten Panel-Analyse zu kombinieren. Als Datengrundlage kommen bei allen drei Verfahren sowohl aggregierte Makro-Daten als auch Individualdaten in Frage.

Gemäss heutigem Stand der Forschung überwiegt die Ansicht, dass die öffentliche Finanzierung von Bildung die Bildungsnachfrage tatsächlich positiv beeinflusst, bzw. dass private Studiengebühren die Bildungsnachfrage schmälern. Unterstützung findet diese These in einer ganzen Reihe von Zeitreihenund Querschnittsuntersuchungen (Dynarsky, 1999; Fredricksson, 1997; Huijsman, Kloek, Kodde \& Ritzen, 1986; Kane, 1995; McPherson \& Schapiro, 1991; Winter-Ebmer \& Wirz, 2002). Allerdings gehen die Einschätzungen über die relative Bedeutung dieser Effekte teilweise relativ weit auseinander und es gibt es auch Untersuchungen, die keinen oder lediglich einen schwachen Einfluss öffentlicher Finanzierung auf die Bildungsbeteiligung finden (Card \& Lemieux, 2000; McPherson \& Schapiro, 1991).

Verschiedene Untersuchungen zeigen auch, dass die Wirkung öffentlicher Finanzierung nicht auf alle Individuen und Haushalte gleich gross ist. Verschiedene Studien weisen nach, dass öffentliche Unterstützung vor allem bei ärmeren Haushalten die gewünschte Wirkung zeigt, während sie das Verhalten reicherer Haushalte kaum beeinflusst (Dynarsky, 1999; Kane, 1995; McPherson \& Schapiro, 1991).

Eng verbunden mit letzterer Problematik ist auch die Frage nach der Rolle des Haushaltseinkommens der Eltern auf die Bildungsbeteiligung ihrer Kinder. Während der positive Zusammenhang zwischen Haushaltseinkommen und Bildungsbeteiligung empirisch unbestritten und in der Literatur hinreichend belegt ist, gibt es für die Ursache dieses Zusammenhangs heute unterschiedliche Interpretationen. Wie neuere Untersuchungen für die USA und Grossbritannien zeigen, verschwindet bzw. verringert sich der Einfluss des Haushaltseinkommens auf die Bildungsnachfrage, wenn man den Einfluss des Familienhintergrundes (bspw. der Bildung der Eltern) und des Intelligenzquotienten des Kindes berücksichtigt, womit die Wirkung einfacher finanzieller Transfers zur Förderung der 
Bildungsbeteiligung zumindest teilweise in Frage gestellt werden kann. Insbesondere könnten bspw. Humankapitalinvestitionen in früheren Jahren (beispielsweise in der Vorschule oder während der obligatorischen Schulzeit) effektiver sein (Cameron \& Heckmann, 1998; Chevalier \& Lanot, 2002).

Zusammenfassend lässt sich dennoch sagen, dass eine Privatisierung von Bildungskosten mit hoher Wahrscheinlichkeit einen Rückgang der Bildungsbeteiligung zur Folge hat, wobei mit einen Rückgang der Nachfrage am ehesten bei ärmeren Haushalten zu rechnen wäre. Umgekehrt gesagt, ist davon auszugehen, dass die Bildungsnachfrage durch öffentliche Finanzierung gesteigert werden kann. Zu ungenau sind die Studien allerdings, um hier eine Aussage darüber zu machen, wie gross die Elastizität der Nachfrage, also der Nachfragerückgang in Abhängigkeit einer bestimmten Reduktion an öffentlicher Unterstützung, wäre.

\section{Qualifikationsangebot, -nachfrage und relative Löhne}

Gemäss unserem in Abbildung 3 dargestellten Modell hat ein Rückgang der Bildungsnachfrage eine Verknappung des Qualifikationsangebots auf dem Arbeitsmarkt zur Folge, was sich in einer steigenden Bildungsprämie niederschlägt. Der Zusammenhang zwischen Angebot- und Nachfrage nach Qualifikationen und den Reaktionen auf dem Arbeitsmarkt ist in der ökonomischen Literatur sehr gut dokumentiert (Acemoglu, 2001; Gotschalk, 1997; Johnson, 1997; Topel, 1997). Die Vorstellung, wonach eine Verknappung von Qualifikationen die relativen Löhne qualifizierter Arbeitskräfte steigert, ist dabei unbestritten. Allerdings erweist es sich auch heute als schwierig, Veränderungen in den relativen Löhnen eindeutig entweder auf Veränderungen der Nachfrage und/oder des Angebotes an Qualifikationen zurückzuführen.

Eine zentrale Rolle für die Entwicklung der Qualifikationsnachfrage spielt der technologische Fortschritt, welcher die Nachfrage nach bestimmten Qualifikationen je nach Ausrichtung dämpfen oder steigern kann. Die letzten dreissig Jahre waren durch einen technologischen Fortschritt geprägt, welcher die Nachfrage nach qualifizierten Arbeitskräften stark gesteigert und wenig qualifizierte Arbeit substituiert hat (sog. «skill-biased technological change») und die meisten Experten gehen auch davon aus, dass sich dieser Trend weiter fortsetzen wird.

Auf Seiten des Qualifikationsangebots stehen Humankapitalinvestitionen sicherlich im Vordergrund des Interesses. Die Art der Bildungsfinanzierung spielt für die Entwicklung des Angebots an Qualifikationen offensichtlich eine Rolle, wie in diesem Beitrag gezeigt wurde. Ebenso wichtig dürfte aber die (erwartete) Entwicklung der Lohnprämie sein, welche Schulabgängerinnen und -abgänger erwarten können. Daneben ist aber auch zu berücksichtigen, dass das Qualifikationsangebot nicht nur durch die Bildungsnachfrage, sondern beispielsweise auch durch Veränderungen im Partizipationsverhalten, durch Variation der Arbeitszeit oder durch Migration beeinflusst werden kann. Je grösser und flexibler solche Potentiale zur Erhöhung bzw. Senkung des Arbeitsangebots sind, desto elastischer wird das Qualifikationsangebot, welches in unserem Marktmodell ve- 
reinfacht als vollkommen inelastisch dargestellt ist. Im Falle der Schweiz spielt die Migration eine grosse Rolle. Während in der Vergangenheit durch Zuwaderung vor allem die Nachfrage nach wenig qualifizierten, billigen Arbeitskräften befriedigt wurde, steht heute die Zuwanderung zur Deckung des wachsenden Bedarfs an spezialisierten und hoch qualifizierten Arbeitskräften im Vordergrund des Interesses (vgl. Sheldon, $2000 \mathrm{zu}$ den Auswirkungen der Zuwanderung in die Schweiz auf relative Löhne).

\section{Bildungsrenditen und Bildungsnachfrage}

Über den Einfluss von Angebot und Nachfrage nach Qualifikationen auf die relativen Löhne, schliesst sich der Kreis unseres Modells, sofern diese in Form von Bildungsrenditen eine Rückwirkung auf die Bildungsnachfrage haben. Da die Erträge von Humankapitalinvestitionen aus der Sicht eines Investors in der Zukunft anfallen, müssen Investitionsentscheide auf der Basis von Erwartungen über zukünftige Löhne getroffen werden. Dass Studierende in der Regel positive Bildungsrenditen erwarten, belegen verschiedene Studien (Brunello, Lucifora \& Winter-Ebmer, 2001; Wolter, 2000). Sofern zwischen effektiven und erwarteten Löhnen ein hinreichend enger Zusammenhang besteht, sollte zudem die gegenwärtige Lohnstruktur gemäss Humankapitaltheorie auch künftige Bildungsentscheide beeinflussen. Die Hypothese, wonach Bildungsrenditen bzw. relative Lohndifferenzen die Bildungsnachfrage beeinflussen, ist in der Literatur häufig getestet worden. Verschiedene, teilweise bereits zuvor zitierte Studien zeigen denn auch einen positiven Zusammenhang zwischen Bildungsrenditen bzw. qualifikationsspezifischen Lohndifferentialen und der Bildungsnachfrage auf (Card \& Lemieux, 2000; Fredricksson, 1997; Lauer, 2002; McPherson \& Schapiro, 1991; Whitfield \& Wilson, 1990; Wilson, Wolfe \& Haveman, 2000).

\section{Zusammenfassung der empirischen Ergebnisse}

Wie die Simulationsberechnungen zeigen, übt das System der Bildungsfinanzierung einen direkten Einfluss auf die Höhe privater und fiskalischer Bildungsrenditen aus. Eine Privatisierung direkter Ausbildungskosten führt - ceteris paribus - zu einer Minderung privater und einer Steigerung fiskalischer Bildungsrenditen. Je nach dem, welche relative Bedeutung direkte Ausbildungskosten im Vergleich zu Opportunitätskosten haben, kann eine Privatisierung von Ausbildungskosten erhebliche Auswirkungen auf Bildungsrenditen haben.

Die heute verfügbare empirische Evidenz lässt den Schluss zu, dass eine stärkere Privatisierung der Bildungsfinanzierung auch tatsächlich zu einer Verminderung der Bildungsnachfrage führt, bzw. dass sich die Bildungsbeteiligung durch zusätzliche öffentliche Mittel steigern lässt. Allerdings gibt es auch Evidenz dafür, dass diese Unterstützung beispielsweise nicht in allen Einkommensklassen gleich wirkt. Die Reaktion der Nachfrage auf eine öffentliche Unterstützung dürfte daher stark davon abhängen, in welcher Form und an wen sie ausgerichtet wird. 
Wie sich ein Rückgang des Qualifikationsangebots auf die QualifikationsLohnprämie auswirkt ist stark vom Kontext, bzw. den Arbeitsmarktbedingungen abhängig. Wird der Rückgang des Qualifikationsangebots bspw. durch Zuwanderung oder durch eine Mobilisierung von nicht- oder nur teilzeiterwerbstätigen Personen kompensiert, fällt der Effekt auf die relativen Löhne und damit auf die Bildungsrenditen geringer aus. Denkbar ist auch, dass als Folge des sich verringernden Angebots auch die Nachfrage sinkt, bzw. weniger stark ansteigt, weil sich bspw. weniger Unternehmen mit hohem Bedarf an qualifizierten Arbeitskräften ansiedeln. Je elastischer Angebot und Nachfrage nach Qualifikationen sind, desto geringer fallen Reaktionen der relativen Löhne und damit der Bildungsrenditen aus.

Welche Rückwirkungen allfällige Reaktionen relativer Löhne schliesslich wieder auf die Bildungsnachfrage haben, hängt davon ab, welche Bedeutung Renditeüberlegungen bei Bildungsentscheiden zukommen. Die heute verfügbare Evidenz zu diesem Thema deutet klar darauf hin, dass Bildungsrenditen bei der Erwartungsbildung und auch bei Bildungsentscheiden eine Rolle zukommt. Allerdings dürfte diese Rückwirkung mit einiger Verzögerung erfolgen, und wohl nur dann relevant sein, wenn tatsächlich spürbare Veränderungen auftreten, welche die Erwartungen potentieller Bildungsnachfrager zu beeinflussen vermögen.

Direkte empirische Evidenz über den Zusammenhang zwischen Bildungsfinanzierung und Bildungsrenditen gibt es heute praktisch nicht. Zwar gibt es, wie zuvor erwähnt, eine ausgiebige Literatur, welche die Entwicklung von Lohnungleichheiten (vor allem im Zeitablauf) untersucht. Daneben wurde schon verschiedentlich versucht, Unterschiede in Bildungsrenditen zwischen verschiedenen Ländern zu erklären (Denny, Harmon \& Lydon, 2002; Trostel, Walker \& Woolley, 2002; Weber, 2002). Institutionelle Unterschiede im Bildungssystem wie bspw. die Art der Finanzierung, wurden dabei allerdings nicht systematisch untersucht. Die hier gemachten Überlegungen zeigen, dass es durchaus sinnvoll wäre, die bildungsökonomische Forschung in dieser Richtung weiter zu treiben, denn die empirische Evidenz zeigt, dass die Bildungsfinanzierung für die Entwicklung des Qualifikationsangebots eine potentiell wichtige Rolle spielt. Die These, wonach eine stärker öffentliche Finanzierung zu einer Verringerung sozialer Bildungsrenditen führt, wäre durchaus einen Test wert.

\section{Schlussfolgerungen}

Die Frage, ob eine Ausbildung privat oder öffentlich finanziert wird, hat einen unmittelbaren Einfluss auf die Höhe privater und fiskalischer Bildungsrenditen. Eine Privatisierung der Bildungsfinanzierung führt - ceteris paribus - zu einer Verringerung privater und einer Erhöhung fiskalischer Bildungsrenditen. Soziale Bildungsrenditen sind von Veränderungen der Finanzierung nicht betroffen, solange sämtliche übrige Einflüsse konstant bleiben. 
Wie die Berechnungen für die Schweiz zeigen, würden private Bildungsrenditen bei einer vollständigen Privatisierung von Ausbildungskosten deutlich unter das Niveau sozialer Bildungsrenditen fallen. In diesem Fall wären die privaten Anreize zur Bildungsnachfrage wohl zu gering, um ein aus gesamtwirtschaflicher Sicht optimales Investitionsniveau zu erreichen. Dies gilt insbesondere, weil Bildungsinvestitionen aus individueller Sicht mit Risiken behaftet sind, welche die Gesellschaft als Ganzes durchaus tragen, bzw. ausgleichen könnte. Soziale und fiskalische Bildungsrenditen weisen in der Schweiz ein respektables Niveau auf, welches in einer ähnlichen Grössenordnung wie in anderen OECD-Ländern liegt, womit Bildung auch aus gesamtwirtschaftlicher Sicht als lohnende Investitionsmöglichkeit anzusehen ist.

Eine wichtige Erkenntnis aus der komparativ statischen Betrachtung ist, dass zwischen dem Steuersystem und dem System der Bildungsfinanzierung ein sehr enger Zusammenhang besteht. Eine öffentliche Bildungsfinanzierung kann sogar als Gegenstück zu einem progressiven Steuersystem angesehen werden. Während letzteres einer impliziten Besteuerung von Humankapitalinvestitionen entspricht, kann diese Verzerrung durch eine öffentliche, steuerliche Bildungsfinanzierung ausgeglichen werden. Wie die positiven fiskalischen Bildungsrenditen zeigen, scheint dieses Gleichgewicht in der Schweiz recht gut erreicht zu sein, da fiskalische Bildungsrenditen in einer ähnlichen Grössenordnung liegen wie der Zinssatz für Staatsanleihen. Der Staat kann also die öffentlichen Bildungsausgaben über sein progressives Steuersystem später wieder hereinholen. Einzig die weitgehend privat finanzierten höheren Berufsausbildungen werden durch das progressive Steuersystem belastet, da hier die öffentliche Subventionierung deutlich geringer ist als in anderen Bildungsbereichen. Ein progressives Steuersystem besteuert eben nicht nur öffentlich, sondern auch privat finanzierte Bildung, oder andere Faktoren, wie bspw. angeborene Fähigkeiten, Fleiss und Arbeitsmotivation, welche den Lohn ebenfalls positiv beeinflussen können.

Bezüglich dynamischer Wirkungen gibt es aus der ökonomischen Literatur viele Untersuchungen zu einzelnen Teilfragen. So weiss man aus verschiedenen Studien, dass eine Privatisierung von Ausbildungskosten mit relativ hoher Wahrscheinlichkeit zu einem Rückgang der Bildungsnachfrage führt. Ein solcher Rückgang führt auf dem Arbeitsmarkt zu einer Verknappung des Qualifikationsangebotes und treibt die Lohnprämie für Qualifikationen in die Höhe, wobei das Ausmass dieser Erhöhung entscheidend von der Elastizität der Arbeitskräftenachfrage und der Flexibiltiät des Angebots (beispielsweise über Migration oder zusätzliche Arbeitszeit) abhängt. Soziale, fiskalische und private Bildungsrenditen steigen als Folge davon an. Sobald diese Entwicklungen wahrgenommen und in die Erwartungsbildung von Bildungsnachfragern eingeflossen sind, steigen die erwarteten Bildungsrenditen wieder leicht an.

Aus diesen Zusammenhängen, welche einzeln empirisch mehrfach getestet wurden, lässt sich die Hypothese ableiten, dass eine stärkere öffentliche Bildungsfinanzierung unter sonst gleichen Voraussetzungen zu einer höheren Bildungs- 
nachfrage und damit zu geringeren sozialen und fiskalischen Bildungsrenditen führen sollte. Allerdings wurde diese These bislang empirisch nie in dieser Form getestet. Wie die Überlegungen dieses Aufsatzes gezeigt haben, wären dabei auch sehr viele weitere Faktoren zu berücksichtigen, welche den geschilderten Zusammenhang überlagern könnten.

Die Liste der aktuell untersuchten Themen im Bereich Bildungsrenditen, Bildungsnachfrage und Bildungsfinanzierung ist sehr lang. Die folgenden, teilweise offenen Fragestellungen werden heute neben anderen sehr intensiv behandelt:

Im Bereich Bildungsrenditen:

- Wie kann der kausale Effekt von Bildung adäquat gemessen werden?

- Wie hoch sind die Bildungsrenditen von Personen, welche heute keine Bildung nachfragen (marginale Bildungsrenditen)?

- Mit welcher Art von Externalitäten sind Bildungsinvestitionen verbunden und wie bedeutend sind sie?

- Gibt es eine optimale Höhe von Bildungsrenditen und wie hoch liegt diese?

Im Bereich Bildungsfinanzierung:

- Welche Rolle spielen Ausmass und Art der öffentlichen Finanzierung für die Bildungsbeteiligung insgesamt und von verschiedenen gesellschaftlichen Gruppen?

- Welches sind die Vor- und Nachteile unterschiedlicher Systeme der Bildungsfinanzierung in Bezug auf Umverteilung und Anreize für Humankapitalinvestitionen?

- Welches sind die Umverteilungswirkungen aktueller Bildungsfinanzierungssysteme?

Gleichzeitig wachsen auch die Anstrengungen, die Erkenntnisse aus den verschiedenen Bereichen vermehrt in einen Zusammenhang zu stellen. Mit der zunehmenden Verfügbarkeit international vergleichbarer Datensätze und neuer ökonometrischen Methoden werden die Erkenntnisse in diesem Gebiet in den nächsten Jahren sicherlich schnell wachsen. Allerdings haben die Überlegungen dieses Aufsatzes auch gezeigt, dass sehr viele Faktoren den Zusammenhang zwischen einem System der Bildungsfinanzierung und den Bildungsrenditen beeinflussen können. Dementsprechend komplex ist denn auch ein Modell, welches diesen Zusammenhang erklären möchte. Häufig ist zudem die Varianz der Bildungsfinanzierungssysteme über die Zeit oder auch zwischen Ländern nicht allzu gross, womit es relativ schwierig ist, Unterschiede in der Wirkung zu messen. Dennoch bleibt die Frage, wie Unterschiede in Bildungsrenditen zwischen Ländern und Veränderungen über die Zeit erklärt werden können, und welche Rolle die Bildungsfinanzierung dabei spielt, eine der grossen offenen und wichtigen Fragen der bildungsökonomischen Grundlagenforschung. 


\section{Anmerkungen}

1 «Externe Effekte» oder «Externalitäten» sind Auswirkungen ökonomischen Handelns auf unbeteiligte Dritte, ohne dass jemand dafür bezahlt oder einen Ausgleich erhält. Bei der Bildung entstehen für die Gesellschaft positive Externalitäten bspw. in Form einer tieferen Kriminalitätsrate, einer geringeren individuellen Beanspruchung von Sozialhilfe oder einer positiven Wirkung auf das Wirtschaftswachstum.

2 Die klassische Aussenwirtschaftstheorie sagt beispielsweise voraus, dass sich Länder bei der Herstellung handelbarer Güter auf diejenige Produktion spezialisieren, welche die relativ gesehen reichlich verfügbaren Produktionsfaktoren (z.B. Humankapital) intensiv nutzt, wenn die Produktionsfaktoren nicht beliebig mobil sind (sog. Heckscher-Ohlin Theorem). Ein grosses Angebot an qualifizierten Arbeitskräften zieht damit Unternehmen an, welche viel Humankapital benötigen.

3 Mit dem Konzept der Lohnelastizitäten beschreibt man in der Ökonomie, wie stark Arbeitsangebot und -nachfrage auf Lohnänderungen reagieren. Von einem vollkommen inelastischen Arbeitsangebot spricht man, wenn die angebotene Arbeitsmenge fix und vom Lohn unabhängig ist (vertikale Angebotskurve). Ein elastisches Arbeitsangebot bedeutet dagegen, dass durch die Erhöhung des Lohnes eine Ausdehnung des Arbeitsangebots bewirkt werden kann.

4 Bei den direkten privaten Kosten wurden folgende Elemente in die Überlegungen einbezogen: Lehrlings-lohn von netto CHF 7'200 pro Jahr bei BA (negative Kosten), direkte Kosten in Form von Ausgaben für Schulgebühren, Schulmaterial und Transportkosten. Die Zahlen stellen eigene, sehr grobe Schätzungen dar.

Zur Schätzung der direkten, öffentlichen Kosten wurden verschiedene Quellen benutzt. BA/HBA: Pricewater-house Coopers (2002); MAT/FHS: Bildungsindikatoren des BFS; UNI: Interkantonale Universitätsvereinbarung 1997. Der Wert für Fachhochschulen gemäss BFS (CHF 37'382) wurde auf CHF 30'000 reduziert, da Fachhochschulen auch Forschung betreiben. Gemäss interkantonaler Universitätsvereinbarung entrichten die Entsendekantone von Studierenden den Universitätskantonen die Kosten der Lehre. Im Durchschnitt betrug dieser Betrag 2002 rund CHF 17`000 pro Jahr (zwischen CHF 9'500 und 46'000, je nach Studienfachgruppe). Dieser Betrag wurde etwas angehoben, da die Beiträge der Entsendekantone nicht ganz kostendeckend sein dürften.

\section{Literatur}

Acemoglu, D. (2001). Technical Change, Inequality, and the Labor Market. Journal of Economic Literature, 40, (1), 7-72.

Alsalam, N. \& Conley, R. (1995). The Rate of Return to Education: A Proposal for an Indicator. Education and Employment. Paris: OECD.

Becker, G.S. \& Mulligan, C.B. (1997). The Endogenous Determination of Time Preference. The Quarterly Journal of Economics, 112, (3), 729-758.

Blaug, M. (1970). An Introduction to the Economics of Education. London: Alan Lane The Penguin Press.

Blöndal, S., Feld, S. \& Girouard, N. (2002). Investment in Human Capital through UpperSecondary and Tertiary Education. OECD Economic Studies, 2002/1, (34). Paris: OECD.

Blundell, R., Dearden, L. \& Sianesi, B. (2001). Estimating the Returns to Education: Models, Methods and Results. CEE-Discussion Paper 16, Centre for the Economics of Education, London.

Bovenberg, A.L. \& Jacobs, B. (2001). Redistribution and Education Subsidies are Siamese Twins. CentER-Discussion Paper 2001-82, Erasmus University. 
Brunello, G., Lucifora, C. \& Winter-Ebmer, R. (2001). The Wage Expectations of European College Students. IZA-Discussion Paper 299.

Bundesamt für Statistik (2002). Die Schweizerische Arbeitskräfteerhebung (SAKE). Neuchâtel: BfS.

Cameron, S.V. \& Heckman, J.J. (1998). Life Cycle Schooling and Dynamic Selection Bias: Models and Evidence for Five Cohorts of American Males. Journal of Political Economy, $106,(2), 262-333$.

Card, D. (1999). The Causal Effect of Education on Earnings. In. O. Ashenfelter \& D. Card (Eds.) (1999). Handbook of Labour Economics, Vol. 3A, (1801-1863). Amsterdam: Elsevier.

Card, D. \& Lemieux, T. (2000). Dropout and Enrolment Trends in the Post-War Period: What went wrong in the 1970s? NBER Working Paper 7658.

Creedy, J. (1994). Financing Higher Education: Public Choice and Social Welfare. Fiscal Studies, 15, (3), 87-108.

Chevalier, A. \& Lanot, G. (2002). The Relative Effect of Family Characteristics and Financial Situation on Educational Achievement. Education Economics, 10, (2), 165-181.

Denny, K., Harmon, C. \& Lydon, R. (2002). Cross-country Evidence on the Returns to Education: Patterns and Explanations. CEPR-Discussion Paper 3199, Centre for Economic Policy Research.

Dur, R.A.J. \& Teulings, C.N. (2001). Education and Efficient Redistribution. Tinebergen Institute Discussion Paper, 2001-090/3.

Dynarski, S.M. (1999). Does Aid matter? Measuring the Effect of Student Aid on College Attendance and Completion. NBER Working Paper 7422.

Eidgenössische Steuerverwaltung (1999). Steuerbelastung in der Schweiz 1998. Bern: Bundesamt für Statistik.

Fredriksson, P. (1997). Economic Incentives and the Demand for Higher Education. Scandinavian Journal of Economics, 99, (1), 129-142.

Gotschalk, P. (1997). Inequality, Income Growth, and Mobility: The Basic Facts. Journal of Economic Perspectives, 11, (2), 21-41.

Harmon, C., Walker, I. \& Westergard-Nielsen, N. (Eds.) (2001). Education and Earnings in Europe: A Cross Country Analysis of the Returns to Education. London: Edward Elgar.

Huijsman, R., Kloek, T., Kodde, D.A. \& Ritzen, J.M.M. (1986). An empirical analysis of college enrollment in The Netherlands. De Economist, 134, 181-190.

Johnson, G.E. (1997). Changes in Earnings Inequality: The Role of Demand Shifts. Journal of Economic Perspectives, 11, (2), 41-54.

Lauer, Ch. (2002). Enrolments in Higher Education: Do Economic Incentives Matter? Education and Training, 44, (4/5), 179-185.

McPherson, M. \& Schapiro, M.O. (1991). Does Student Aid Affect College Enrollment? New Evidence on a Persistent Controvers. American Economic Review, 81, (1), 309-318.

Nagel, B. \& Jaich, R. (2002). Bildungsfinanzierung in Deutschland - Analyse und Gestaltungsvorschläge. Endbericht an die Max-Träger-Stiftung, Frankfurt am Main.

Psacharopoulos, G. (1987). The Cost-Benefit Model. Psacharopoulos, George (Ed.). Economics of Education: Research and Studies. Oxford etc.: Pergamon Press, (S. 342-347).

Pricewaterhouse Coopers (2002). Finanzierung der Berufsbildung. Bericht im Auftrag des Bundesamtes für Berufsbildung und Technologie (BBT). Verfasst von Claudia Strausak und Beat Blaser.

Sheldon, G. (2000). The Impact of Foreign Labor on Relative Wages and Growth in Switzerland. WWZ-Working Paper, University of Basel.

Sianesi, B. \& Van Reenen, J. (2002). The Returns to Education: A Review of the Empirical Macro-Economic Literature. IFS-Working Paper 02/05, Institute of Fiscal Studies, London.

Thurow, L.C. (1975). Generating Inequality. New York: Basic Books.

Topel, R.H. (1997). Factor Proportions and Relative Wages: The Supply-Side Determinants of Wage Inequality. Journal of Economic Perspectives, 11, (2), 55-74. 
Trostel, Ph., Walker, I. \& Woolley, P. (2002). Estimates of the economic return to schooling for 28 countries. Labour Economics, 9, (1), 1-16.

Weber, B.A. (2002). The link between unemployment and returns to education: evidence from 14 European countries. Education and Training, 44, (4/5), 171-178.

Whitfield, K. \& Wilson, R.A. (1991). Staying on in Full-time Education: The Educational Participation Rate of 16-year-olds. Economica, 58, (231), 391-404.

Wilson, K., Wolfe, B. \& Haveman, R. (2000). The Role of Expectations in Adolescent Schooling Choices: Do Youths Respond to Economic Incentives? Paper presented at the 56th Congress of the IIPF: Public Finance and Human Capital, 28-31 August 2000, Seville, Spain.

Winter-Ebmer, R. \& Wirz A. (2002). Public Funding and Enrolment into Higher Education in Europe. IZA-Discussion Paper 503.

Wolter, S.C. (2000). Wage Expectations: A Comparison of Swiss and US Students. Kyklos, 53, (1), 51-69.

Wolter, S.C. (2001). Bildungsfinanzierung zwischen Staat und Markt. Chur \& Zürich: Rüegger.

Wolter, S.C. (2002). Bildungsökonomie - Eine Standortbestimmung. Schweizerische Zeitschrift für Bildungswissenschaften, 24, (1), 149-169.

Wolter, S.C. \& Weber, B.A. (1999). On the Measurement of Private Rates of Return to Education. Jahrbücher für Nationalökonomie und Statistik, 218, (5/6), 605-618.

\section{Financement et rendements de la formation}

\section{Résumé}

L'article traite des liens entre le mode de financement (public vs. privé) et les taux de rendement de l'éducation (TRE). Dans une première section, les notions de la théorie du capital humain et des TRE privés, sociaux et fiscaux sont définis. La deuxième partie décrit les interactions entre les modes de financement et les TRE de manière comparativo-statique ainsi que dynamique. Dans une troisième partie, des estimations de TRE privés, fiscaux et sociaux sont présentées pour le cas des hommes en Suisse. Sur la base de ces estimations - avec un financement mixte public et privé - l'impact d'un financement purement public et privé sur les TRE est simulé de manière comparativo-statique. A l'aide d'études empiriques internationales, les réactions dynamiques potentielles sont estimées. L'article conclut en esquissant des directions possibles de recherche, pour mieux comprendre les relations entre les modes de financement et les TRE. 


\section{Finanziamento e rendita della formazione}

\section{Riassunto}

Il contributo affronta la questione della relazione tra finanziamento (pubblico e privato) e rendita della formazione. Inizialmente si propone una breve introduzione alle basi della teoria del capitale umano e si espongono i concetti della rendita privata, fiscale e pubblica. In un secondo capitolo si discute il rapporto teorico tra finanziamento e rendita della formazione facendo riferimento ad aspetti sia statico-comparativi sia dinamici. Il terzo capitolo è dedicato alla presentazione di stime della rendite formative privata, fiscale e sociale per uomini in Svizzera. Questi dati permettono di illustrare le conseguenze che i due modelli di finanziamento, completamento privatizzato da un lato oppure completamente pubblico dall'altro, avrebbero sulla rendita formativa. Si aggiungono poi riflessioni di carattere dinamico basate su dati empirci internazionali. L'articolo conclude con una panoramica sui campi di ricerca attuali e di possibile rilevanza futura.

\section{Funding and Returns to Education}

\section{Summary}

The article deals with the various links between public or private funding and rates of return to education (RRE). In a first section the notion of human capital theory as well as the notions of private, social and fiscal RRE's are defined. The second part is devoted to exploring the interaction between financing modes and RRE's in a comparative-static as well as in a dynamic setting. In the third part estimates of private, fiscal and social RRE's are presented for the case of men in Switzerland. On the basis of these results - with mixed public and private funding - the impact of a totally public and private funding of direct educational costs on RRE's is simulated in a comparative-static manner. An estimate on dynamic reactions is made with the help of international empirical results. The article ends with conclusions on new directions to take to further investigate the relationship between financing modes and RRE's. 